\title{
PONTAS EVOCADAS POR ESTÍMULOS SOMATOSSENSITIVOS E ATIVIDADE EPILEPTIFORME NO ELETRENCEFALOGRAMA EM CRIANÇAS "NORMAIS"
}

\author{
Lineu C. Fonseca', Glória M. A. S. Tedrus ${ }^{1}$
}

\begin{abstract}
RESUMO - Estudamos a ocorrência de potenciais de alta voltagem evocados por estímulos somatossensitivos pontas evocadas (PE) - e de atividade epileptiforme espontânea (AE) no EEG de 173 crianças normais de 7 a 11 anos de idade. Durante o EEG, dez percussões foram realizadas nas mãos e pés. Foi avaliada a ocorrência de $P E$ acompanhando cada um dos estímulos e a presença de $A E$. AE foi observada em quatro crianças (2,3\%): pontas centroparietais em duas, complexos de ponta-onda lenta generalizados em uma e pontas parietais e temporais médias em uma. Uma menina de 10 anos de idade $(0,58 \%)$ teve ao EEG pontas parietais medianas evocadas pela percussão do pé esquerdo. Este EEG era normal quanto a outros aspectos. Nossos achados de AE em crianças normais são similares aos encontrados em estudos de outros países. Constatamos que espículas somatossensitivas podem ser observadas em crianças normais o que sugere uma natureza funcional ligada à idade.
\end{abstract}

PALAVRAS-CHAVE: eletrencefalograma, pontas evocadas, atividade epileptiforme, crianças normais.

\section{Somatosensory evoked spikes and epileptiform activity in "normal" children}

\begin{abstract}
Little is known about somatosensory evoked spikes (SES) in the EEG of normal children. We studied the occurrence of SES and spontaneous epileptiform activity (SEA) in 173 normal children ageg 7 to 11 years. During the EEG ten taps were applied to both hands and feet. The occurrence of high voltage potentials evoked by each stimulation of one or both heels or hands (SES) and the occurrence of SEA were evaluated. SEA was observed in four children (2.3\%): central/parietal spikes in two cases, generalized spikeand-wave in one, and parietal/midtemporal spikes in one case. A ten years old girl $(0,58 \%)$ had SES on median parietal region by tapping the left foot. This EEG was otherwise normal. Our findings of SEA are similar to those obtained in other normal populations. SES can be observed in normal children. These SES suggest that we are dealing with an age-related functional phenomenon.
\end{abstract}

KEY WORDS: evoked spikes, electroencephalogram, normal children, epileptiform activity

A publicação da Liga Internacional Contra Epilepsia sobre Classificação e Terminologia ${ }^{1}$ destaca o grupo das epilepsias idiopáticas focais da infância e aponta três tipos: epilepsia benigna da infância com pontas centrotemporais, epilepsia occipital benigna da infância de início precoce (tipo Panayiotopoulos) e epilepsia idiopática occipital de início tardio (tipo Gastaut). Nessas síndromes não existem lesões anatômicas demonstráveis e há remissão espontânea das crises. As crises epilépticas e os achados eletrencefalográficos são focais. As crianças não apresentam déficit neurológico ou intelectual e nem antecedentes de doenças do sistema nervoso central. Muitas crianças apresentam antecedentes familiares de epi- lepsia ou crise febril. Os achados clínicos e eletrencefalográficos são fundamentais na caracterização dessas síndromes ${ }^{2}$. Atividade epileptiforme semelhante à observada nessas síndromes epilépticas benignas da infância tem sido observada em cerca de 3\% das crianças de estudos populacionais normais ${ }^{3-5}$. No Brasil, temos conhecimento de apenas um estudo de atividade epileptiforme em crianças normais, na faixa etária de 4 a 8 anos de idade ${ }^{6}$.

Outras síndromes foram descritas, como a epilepsia benigna com sintomas afetivos ${ }^{7}$. De Marco ${ }^{8}$ descreveu outra possível forma de epilepsia benigna da infância com crises motoras - predominantemente versivas ou hemigeneralizadas. $O$ eletrencefalograma

'Professor Titular de Neurologia da Faculdade de Medicina da Pontifícia Universidade Católica de Campinas (PUC-Campinas), Campinas SP, Brasil.

Recebido 28 Março 2003. Aceito 16 Maio 2003.

Dr. Lineu C Fonseca - Rua Sebastião de Souza 205/122 - 13020-020 Campinas SP - Brasil. 


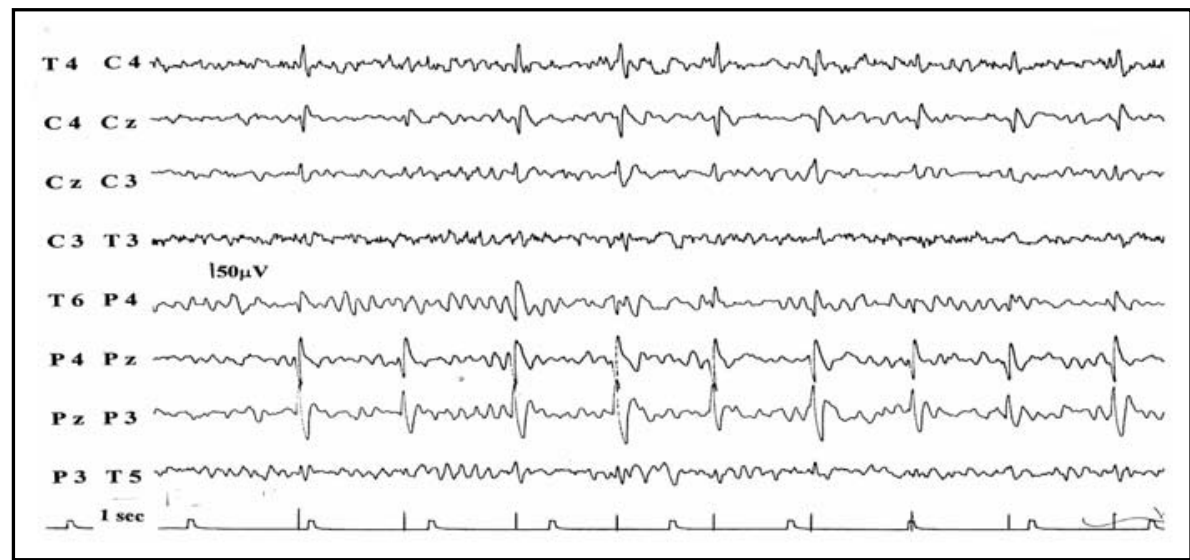

Fig 1. Pontas em Pz e Cz evocadas pela percussão do pé esquerdo.

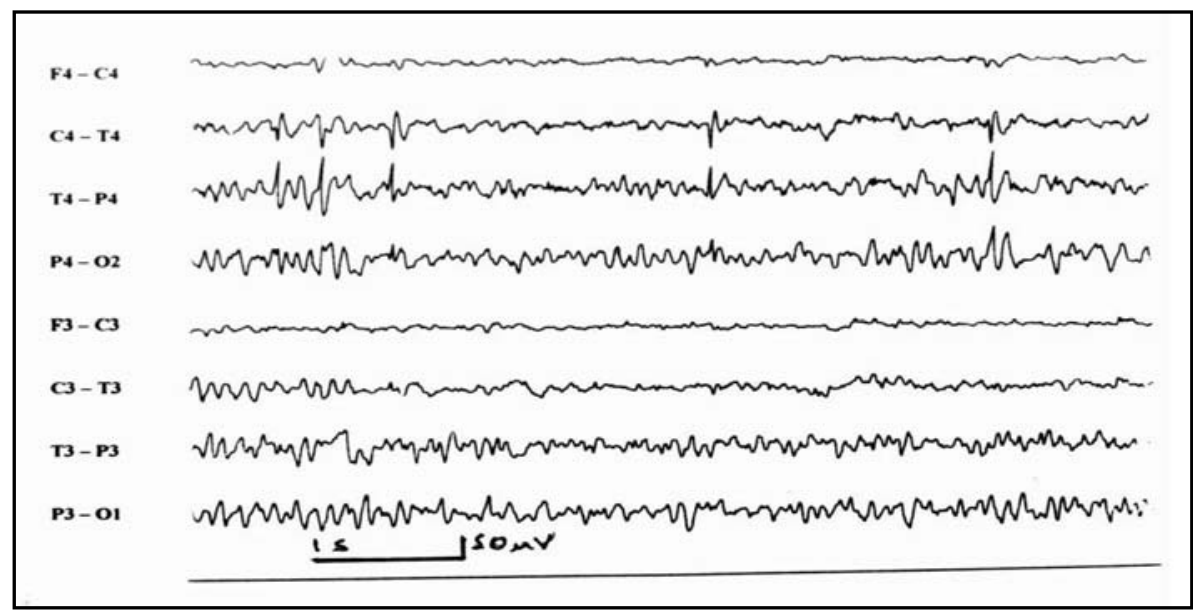

Fig 2. Pontas espontâneas na região temporal média direita

(EEG) mostra pontas parietais evocadas pela percussão dos pés e mãos e atividade de base normal. Essa epilepsia teria evolução em quatro fases: 1 . Entre 2,5 e 5,5 anos de idade, em que as pontas evocadas ocorrreriam como achado isolado ao EEG; 2 . Aparecimento de pontas espontâneas apenas durante o sono; 3. Aparecimento de pontas espontâneas também durante vigília; 4 . Início das crises epilépticas, de 5 a 24 meses após. Predomina no sexo masculino e as crises são pouco freqüentes. Existem controvérsias se essa forma seria uma variante da epilepsia com pontas centrotemporais. Em estudos anteriores mostramos que pontas parietais evocadas por estímulos somatossensitivos (PE) ocorrem em várias síndromes epilépticas mas, na maioria das vezes, em pacientes sem sinais de lesão cerebral| ${ }^{9,10,11}$. Destas crianças sem sinais lesionais, algumas tinham quadro clínico das epilepsias benignas da infância - com pontas centro-temporais ou com paroxismos occipitais - ou das epilepsias generalizadas idiopáticas, mas proporção significativa delas correspondiam ao quadro descrito por De Marco ${ }^{8}$. No entanto, na maioria das vezes as PE ocorrem em crianças sem manifestações epilépticas ${ }^{11}$ Não temos conhecimento de pesquisa em crianças normais.

O objetivo desta pesquisa é estudar a ocorrência de PE e atividade epileptiforme em população de crianças normais.

\section{MÉTODO}

O projeto de pesquisa foi aprovado pelo Comitê de Ética em Pesquisa em Seres Humanos da Pontifícia Universidade Católica de Campinas.

Foram avaliadas 261 crianças provenientes de 4 escolas públicas de Campinas-SP, com idade entre 7 e 11 anos. Cada sujeito passou pelas seguintes avaliações: 1. Anamnese com pais ou responsáveis para levantar antecedentes pessoais, queixas neurológicas, psiquiátricas assim como o desempenho escolar; 2 . Exame neurológico tradicional; 3. Teste de desempenho escolar (TDE) - avaliação das seguintes capacidades fundamentais para o desempenho escolar: escrita, aritmética e leitura; 4 . Teste matrizes progressivas de Raven - avaliação intelectual com rela- 
ção a idade da criança; 5. Inventário de Edinburg: avaliação da dominância lateral; 6 . EEG digital incluindo repouso, hiperpnéia de 3 minutos de duração, 10 percussões de pés e mãos, com martelo de reflexos, com intensidade equivalente à pesquisa de reflexos profundos.

Das 261 crianças, foram incluídas 173 que não apresentavam na anamnese ou na avaliação neurológica ou psicológica evidências de lesão cerebral ou história familiar de epilepsia.

No EEG foi pesquisada a ocorrência de potenciais de alta voltagem evocados acompanhando cada percussão (PE) (Fig 1). Foi também avaliada a presença de atividade epileptiforme (Fig 2).

\section{RESULTADOS}

Foi observada atividade epileptiforme em quatro $(2,3 \%)$ crianças. Esta foi caracterizada por pontas centro-parietais em dois casos e parietais e temporais médias em um caso. Em outro caso ocorreram complexos de ponta-onda generalizados.

Uma menina de 10 anos de idade apresentou pontas parietais medianas evocadas pela percussão do pé esquerdo, no EEG Neste exame não existiam outras anormalidades.

\section{DISCUSSÃO}

$\mathrm{O}$ achado de atividade epileptiforme em 2,3\% das crianças desta pesquisa é equivalente ao observado em crianças suecas $^{4}$, norte-americanas ${ }^{3}$ e japonesas ${ }^{11}$ o que sugere que fatores étnicos não têm importância significativa na gênese da atividade epileptiforme.

Neste estudo constata-se que PE também podem ocorrer em crianças normais.
As PE assim como as pontas espontâneas observadas em crianças normais podem ser interpretadas como elementos de natureza funcional idade-dependente. As PE estão, provavelmente, na maioria dos casos, ligadas a condição benigna ${ }^{11-13}$, envolvendo especialmente o lobo parietal ${ }^{11}$.

A ausência de relatos, na literatura mundial, de PE em crianças normais deve-se provavelmente ao fato de que sua existência não foi objeto de pesquisa sistematizada.

\section{REFERÊNCIAS}

1. Engel J A proposed diagnostic scheme for people with epileptic seizures and with epilepsy: report of the ILAE task force on classification and terminology. Epilepsia 2001;42:1-6.

2. Aicardi J. Epileptic syndromes in childhood. Epilepsia 1988;29:S1-5.

3. Gibbs FA, Gibbs EL. Atlas of electroencephalography, Vol III. Reading: Addison-Wesly, 1964:15.

4. Eeg-Oloffson O, Petersen I, Selden U. The development of the electroencephalogram in normal children from age 1 through 15 years. Neuropadiatrie 1971;2,375-404.

5. Cavazzuti GB, Capella L, Nalin A . Longitudinal study of epileptiform EEG patterns in normal children. Epilepsia 1980;21,43-55.

6. Fonseca LC. Eletrencefalograma em crianças normais de 4 a 8 anos de idade. Tese. Campinas, 1976.

7. Dalla Bernardina B, Bureau M, Dravet C, Dulac O Tassinari CA, Roger J. Epilepsie bénigne de l'enfant avec crises à sémeiologie affective. Rev EEG Neurophysiol 1980,10:8-18

8. De Marco P. Evoked parietal spikes and childhood epilepsy. Arch Neurol 1980;37:291-292

9. Fonseca LC, Tedrus GMA. Epileptic syndromes in children with somatosensory evoked spikes. Clin Electroencephalograph 1994;25:54-58.

10. Fonseca LC; Tedrus GMAS. Epilepsia com pontas centrotemporais e com pontas parietais: estudo comparativo. Arq Neuropsiquiatr 1995;53:281-283

11. Fonseca LC, Tedrus GMAS. Somatosensory evoked spikes and epileptic seizures: a study of 385 cases. Clin Electroencephalograph 2000;31:71-75

12. Okubo $Y$, Matsura M, Asai T, et al. Epileptiforme EEG discharges in healthy children: prevalence, emotional and behavioral correlates, and genetic influences. Epilepsia 1994,35,832-841.

13. Langill L, Wong PKH. Tactile-evoked rolandic discharges: a benign finding? Epilepsia 2003;44:221-227. 